\title{
Pulmonary damage following right ventricular outflow tachycardia ablation in a child: When electroanatomical mapping isn't good enough
}

\author{
Neha Bansal MD (iD | Daisuke Kobayashi MD | Peter P. Karpawich MSc, MD
}

Division of Cardiology, Carman and Ann Adams Department of Pediatrics, Children's Hospital of Michigan, Wayne State University School of Medicine, Detroit, MI, USA

Correspondence

Peter P. Karpawich, MSc, MD, Division of Cardiology, Carman and Ann Adams Department of Pediatrics, Children's Hospital of Michigan, Wayne State University of Medicine, 3901 Beaubien Blvd, Detroit, MI 48201-2119. Email:pkarpawi@dmc.org

\begin{abstract}
A 14-year-old female was referred for severe pulmonary valve insufficiency after undergoing radiofrequency ablation for a right ventricular outflow tract tachycardia that originated in the proximal pulmonary artery at 10 years of age. Clinical records indicated that ablation was guided solely by electrograms and electroanatomical mapping. Due to myocardial tissue extensions, mapping failed to identify the level of the pulmonary valve annulus, which resulted in delivery of energy on the valve proper and into the pulmonary artery. She developed severe pulmonary valve insufficiency and moderate proximal pulmonary artery stenosis necessitating intravascular stent placement 4 years later with an associated transcatheter valve. Although the nonfluoroscopic approach during ablation has gained wide acceptance for use in children, this report highlights the benefits of adjunctive imaging to identify the precise location of the pulmonary valve when ablation therapy is contemplated in the right ventricle outflow tract.
\end{abstract}

KEYWORDS

electroanatomical mapping, nonfluoroscopy ablation, pulmonary artery, radiofrequency ablation, right ventricular outflow tachycardia

\section{1 | INTRODUCTION}

Ventricular tachycardia (VT) is rare in children, especially without underlying cardiac disease. ${ }^{1,2}$ The majority of idiopathic VTs originate in the right ventricular outflow tract (RVOT) and can be effectively treated with radiofrequency (RF) ablation. ${ }^{1,3}$ However, in some circumstances, a unique situation exists in which the arrhythmogenic focus may originate distal to the pulmonary valve. It has been speculated that myocardial remnants in the main pulmonary artery (PA) trunk provide the arrhythmia substrate. ${ }^{4-7}$ To date, there have been no reported major complications associated with RF ablation along the RVOT or within the PA. Although fluoroless ablations eliminate radiation-related concerns and have been shown to be effective in children for some arrhythmias, reliance solely on electroanatomical mapping is not always advisable. We report a case of a child with the diagnosis of an RVOT tachycardia, treated with RF ablation inadvertently applied inside the PA guided by electroanatomical mapping. The procedure resulted in severe pulmonary valve damage and significant proximal PA stenosis requiring intervention.

\section{I CASE REPORT}

A 14-year-old female was referred for severe pulmonary insufficiency and proximal PA stenosis 4 years after ablation for a RVOT tachycardia. From available records, she had a history of frequent premature ventricular contractions (PVCs) starting at 7 years of age. The inferior QRS axis and the left bundle branch block pattern were compatible with an RVOT location (Figure 1A). She was initially treated medically, but after 3 years, symptoms worsened and a 24-hour Holter monitor showed a PVC-burden of more than 35\%. During an exercise stress test, ventricular ectopy continued, culminating in a short runs of VT ( 167 beats/min) associated with dizziness. The initial echo/Doppler study showed normal biventricular systolic function with a normal pulmonary valve without stenosis or insufficiency. Due to the high PVCburden and exercise-induced VT, an electrophysiology/ablation study was performed.

From available records, the electrophysiology mapping was performed using the EnSite system (Endocardial Solutions/St. Jude Medical, St. Paul, MN, USA) and intracardiac electrograms. Programmed stimulation showed normal sinus and atrioventricular nodal 
(A) Baseline

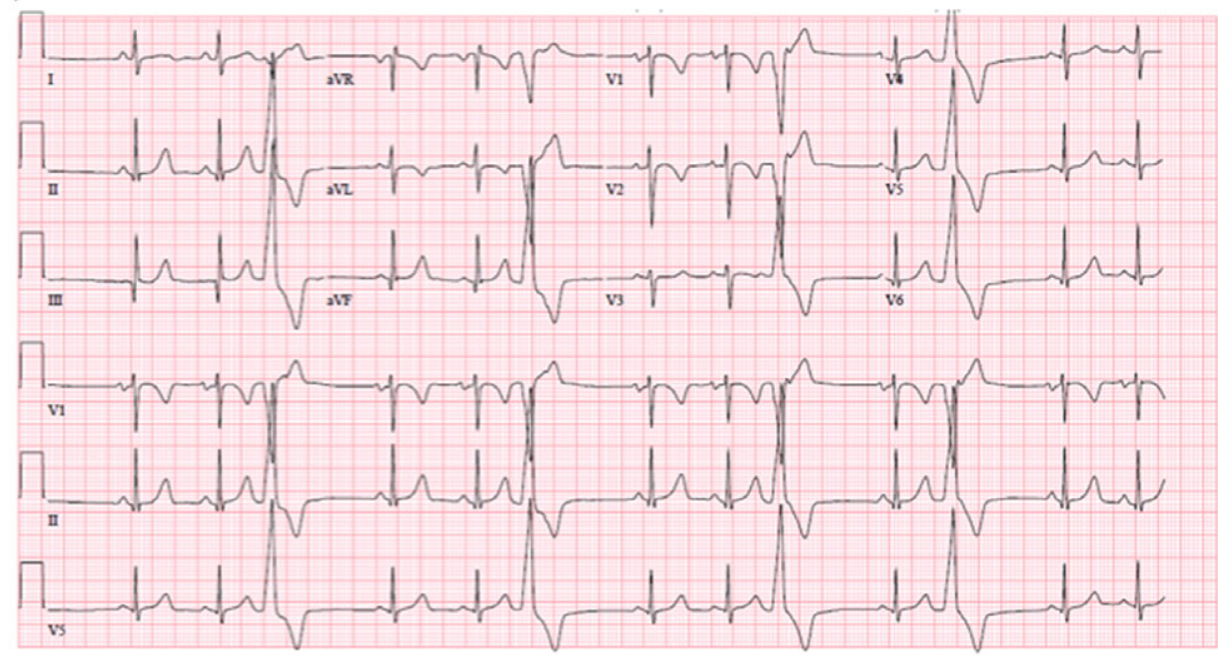

(B) Inducible ventricular tachycardia at Electrophysiology Study

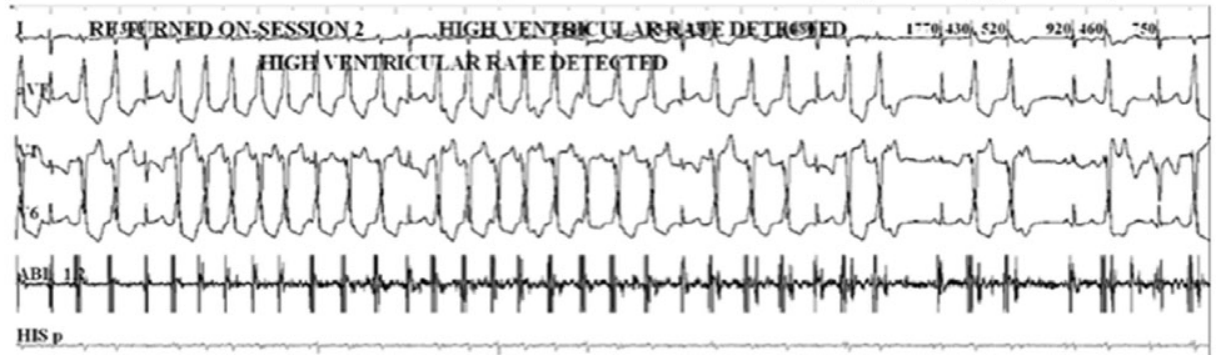
HIs p

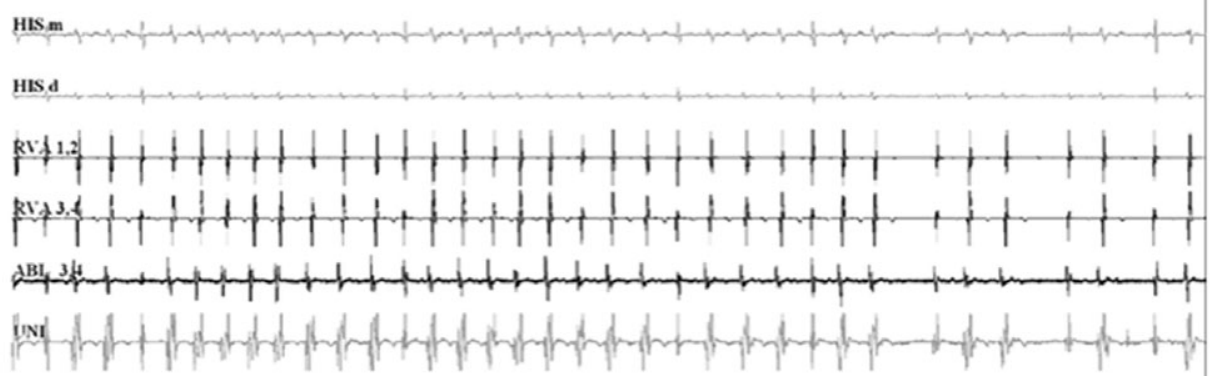

FIGURE 1 A, Baseline 15-lead electrocardiogram, showing frequent monomorphic premature ventricular contractions having an inferior QRS axis and left bundle branch block morphology. B, Intracardiac recording during the electrophysiology study, showing inducible ventricular tachycardia of the same morphology [Color figure can be viewed at wileyonlinelibrary.com]

functions, with no inducible atrial arrhythmias. However, ventricular ectopic complexes and runs of VT were induced (Figure 1B). Left ventricular mapping was unremarkable. Activation mapping revealed the arrhythmia location to be along the presumed distal RVOT, proximal to the valve. Pace-mapping of the region effectively localized the ectopic origin with a 12/12 match. RF ablation was then performed at the mapped focus using an 8-mm-tip Sapphire catheter (St. Jude Medical). Fluoroscopy was not used nor were ventricular/PA angiograms. Concomitant echocardiography was not performed. A total of $14 \mathrm{RF}$ ablations with standard myocardial temperatures between $50^{\circ} \mathrm{C}$ and $70^{\circ} \mathrm{C}$ were delivered with effective elimination of all ectopy (Figure 2). Postablation pacing protocols failed to reinduce any arrhythmias.

The following day, an echocardiography with Doppler study revealed abnormalities of the pulmonary valve and proximal PA with a trace pericardial effusion. The PA appeared narrow and echo- bright at the tip of the pulmonary valve leaflets. Doppler analysis showed pulmonary valve insufficiency. Four years later she remained arrhythmia-free but was referred for an interventional PA stent and valve insertion due to progressive fatigue and shortness of breath (New York Heart Association class II-III). A pre-stent echocardiography with Doppler study showed a dilated right ventricle, stenosis in the proximal PA (peak gradient of $51 \mathrm{~mm} \mathrm{Hg}$ ) and free pulmonary valve insufficiency (Figure 3 ). A PA angiogram performed at the time of the interventional study confirmed the site of ablation-induced narrowing to be distal to the valve.

\section{3 | DISCUSSION}

RVOT tachycardia, in the pediatric population, typically has an excellent prognosis and is amenable to ablation therapy. A study of 


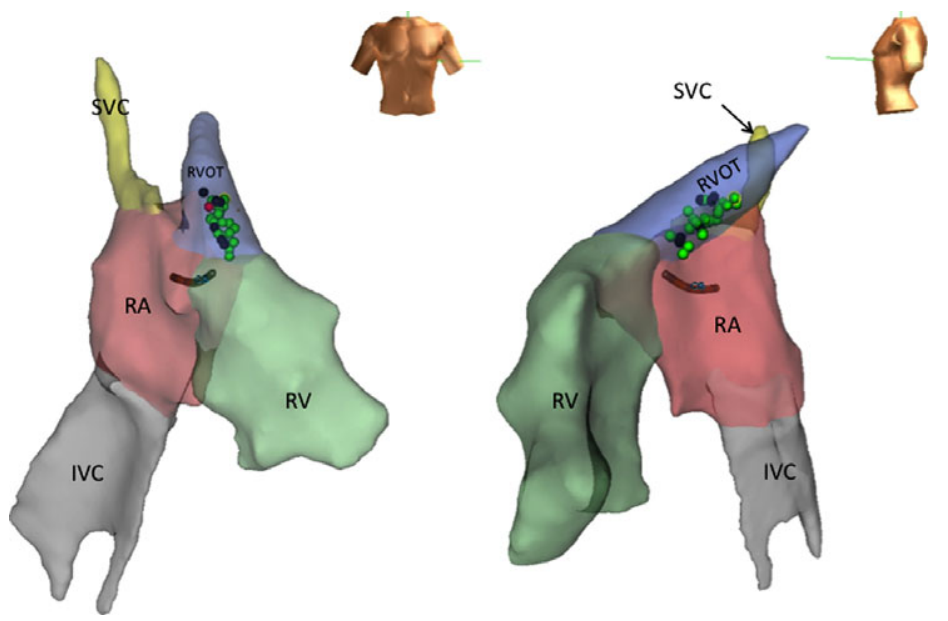

FIGURE 2 Anteroposterior (left) and lateral (right) views of the EnSite ${ }^{\circledR}$ electroanatomical map obtained at the time of ablation with energy delivery demarcated by dots. The region labeled "RVOT" was erroneously thought to be the right ventricular outflow tract based on activation sequences. As can be seen, the ablation was in the proximal pulmonary artery and not the subpulmonary outflow myocardium. IVC = inferior vena cava; RA = right atrium; SVC = superior vena cava; $\mathrm{V}=$ ventricle [Color figure can be viewed at wileyonlinelibrary.com]
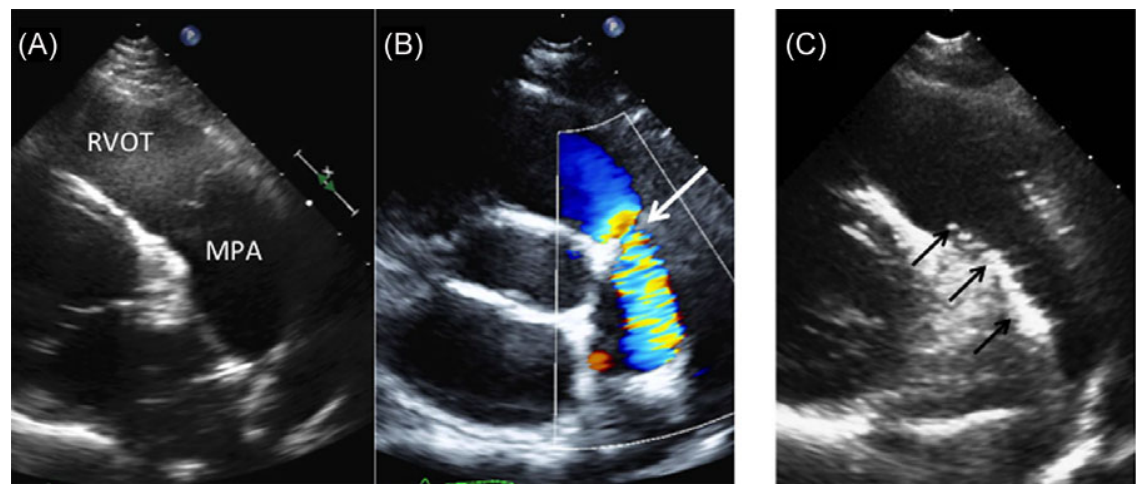

FIGURE 3 Parasternal echocardiographic long axis view showing the RVOT and MPA. A, Initial appearance before ablation, illustrating a normal valve and outflow. B, Comparable view with Doppler taken 4 years later showing increased echogenicity at the valve (arrow) and flow alteration. $\mathrm{C}$, Comparable view showing a stent placed (arrow) in the area of pulmonary stenosis. MPA = main pulmonary artery; RVOT = right ventricular outflow tract [Color figure can be viewed at wileyonlinelibrary.com]

children less than 16 years of age in a large catchment area by Roggen et al. reported only 27 patients with spontaneously occurring episodes of VT (VT incidence of 1.1 episodes/100,000 childhood years). ${ }^{2}$ The tachycardia has been shown to be adrenergically mediated and sensitive to intracellular calcium levels with the substrate mechanism due to cyclic adenosine monophosphate-mediated triggered activity dependent on delayed after depolarizations. ${ }^{8}$ To our knowledge, this report is unique in two aspects: young patient age (10 years old) at the time when ablation was performed in the PA and the rare association of the arrhythmogenic focus localized in the proximal PA due to myocardial extensions. It also highlights a potential complication of RF ablation within the PA and inaccuracies with reliance on electroanatomical mapping.

The mechanism of PA-focus of the "RVOT" arrhythmia is extremely intriguing. Animal studies have shown that the distal myocardial boundary of the outflow tract is not a stable landmark but moves proximally over the spiraling course of the aorta-pulmonary trunk until the valves develop and septation occurs. ${ }^{9}$ The distal myocardial bound- ary of the outflow tract then continues to regress and the conus septum becomes largely incorporated into the right ventricle. ${ }^{9}$ In some individuals, however, there can be incomplete regression resulting in a myocardial sleeve that extends into the PA, which may remain connected to the RVOT. ${ }^{10,11}$ Liu et al. demonstrated that myocardial extension in the PA is, in fact, very common (92\%) even in normal subjects, although this extension does not always cause arrhythmias. ${ }^{11}$ Forty-six percent (11/24 patients of RVOT arrhythmia) had arrhythmic foci located above the pulmonary valve. More recently, the incidence of a PA arrhythmia origin has been reported from $8 \%$ to $33 \%$ among patients with a left bundle branch block morphology VT. ${ }^{11,12}$ It is important to be aware of this possibility of a PA arrhythmia when VT from RVOT is initially suspected.

Nonfluoroscopy techniques have been effective and safe for ablation of cardiac arrhythmias with elimination of radiation exposure, especially in children. Recent meta-analysis showed that ablations performed with zero or near-zero fluoroscopy showed no difference in efficacy and safety. ${ }^{13}$ Recently, ALARA (as-low-as-reasonably 
achievable) protocols using low radiation dose have shown to have markedly reduced radiation exposure, yet providing equivocal ablation efficacy and procedure safety. ${ }^{14}$ However, that may not be the best choice in the RVOT, where electroanatomic mapping may fail to recognize the level of pulmonary valve annulus, typically determined where myocardial voltages suddenly disappear or markedly decrease. In the literature, a cutoff voltage value of $1.9 \mathrm{mV}$ has been used to discriminate between subvalvular and supravalvular pulmonary positions $(90 \%$ sensitivity, $96 \%$ specificity). ${ }^{15}$ However, this is based on adult data and the assumption that myocardial signals terminate precisely at the level of the pulmonic valve annulus. ${ }^{16}$

Previous reports have recommended the use of angiography to locate the ablation catheter in relation to the pulmonary valve for RVOT arrhythmias. 4,5,7 Transthoracic or intracardiac echocardiography (ICE) may provide additional real-time guidance to demonstrate the catheter position and pulmonary valve. ${ }^{11}$ In this present case, as seen in Figure 2, the region originally labeled "RVOT" was actually the proximal PA, ostensibly because electroanatomical mapping failed to identify the pulmonary valve annulus due to persistence of intracardiac signals. Electrophysiological studies and catheter ablations in children do present some difficulties when compared with older adults. With the current equipment, careful studying of the anatomical location of ablation before and during the ablations using imaging modality like short fluoroscopy times, ICE, or angiography as an adjunct to voltage mapping in children is recommended to decrease complications.

Complications associated with RF ablation on PA arrhythmias have not been reported in previous case reports or series. Our report is the first to demonstrate that RF ablation could damage pulmonary valve leaflets and arterial vascular walls when not cautiously performed. In this case, the detrimental effects were detected soon after the procedure and progressed further, leading to eventual referral and intervention. In addition, since the PA is a vascular structure and different from the endocardium of the RVOT, the endothelial vascular lining is potentially more susceptible to ablation-induced tissue damages. Because of this, Sekiguchi et al. has recommended that RF application should be given only for a limited time with the use of lowered RF energy than if used in the RVOT proper. ${ }^{7}$ However, that implies accurate anatomic identification.

In our case, the reason for this complication of the supravalvular stenosis was secondary to the misidentified location of the arrhythmia focus and, therefore, misguided ablations. In addition, it can be argued that an 8-mm catheter tip may not be the most appropriate size to map and ablate with high level of accuracy, especially in the outflow tract of young patients. In addition to this, the high temperature of $70^{\circ} \mathrm{C}$ used in the thin musculature of the PA (even though used with the intention of ablating in the RVOT) as well as the high number of ablations (total number of 14 ablations) were probably the reasons for this unfortunate complication in this patient. It can be hypothesized that this is a failure of interpretation of the electroanatomical mapping. This case demonstrates dramatically that utmost care must be taken if RF ablation is performed near the pulmonary valve annulus, especially in the young. Compulsive mapping is crucial to confirm the location of the ablations to drastically limit the number of RF applications, as the RF energy can be quite destructive in the PAs.

\section{4 | CONCLUSION}

Myocardial extensions can occur into the proximal PA and arrhythmias arising from such a focus can mimic RVOT tachycardia. RF ablation can cause destruction of pulmonary valve leaflets and significant PA stenosis. Before RVOT/PA ablation is performed, it is mandatory to confirm the catheter location in relation to the pulmonary valve by fluoroscopy, angiography, and/or echocardiography. Reliance solely on electroanatomic mapping, although accurately localizing arrhythmia foci and avoiding potential radiation-related issues, does not precisely identify valve position and can be counterproductive as well as potentially detrimental.

\section{CONFLICT OF INTEREST}

None.

\section{ORCID}

Neha Bansal MD (iD http://orcid.org/0000-0001-7026-3058

\section{REFERENCES}

1. Pfammatter JP, Paul T. Idiopathic ventricular tachycardia in infancy and childhood: a multicenter study on clinical profile and outcome. Working Group on Dysrhythmias and Electrophysiology of the Association for European Pediatric Cardiology. J Am Coll Cardiol. 1999;33:2067-2072. PubMed PMID. 10362215. Epub 1999/06/11. Eng.

2. Roggen A, Pavlovic M, Pfammatter JP. Frequency of spontaneous ventricular tachycardia in a pediatric population. Am J Cardiol. 2008;101:852-854. PubMed PMID. 18328852. Epub 2008/03/ 11. Eng.

3. Harris KC, Potts JE, Fournier A, et al. Right ventricular outflow tract tachycardia in children. J Pediatrics. 2006;149:822-826. PubMed PMID. 17137900. Epub 2006/12/02. Eng.

4. Tada H, Tadokoro K, Miyaji K, et al. Idiopathic ventricular arrhythmias arising from the pulmonary artery: prevalence, characteristics, and topography of the arrhythmia origin. Heart Rhythm. 2008;5:419-426. PubMed PMID. 18313601.

5. Timmermans C, Rodriguez LM, Crijns HJ, et al. Idiopathic left bundlebranch block-shaped ventricular tachycardia may originate above the pulmonary valve. Circulation. 2003;108:1960-1967. PubMed PMID. 14530199.

6. Timmermans C, Rodriguez LM, Medeiros A, et al. Radiofrequency catheter ablation of idiopathic ventricular tachycardia originating in the main stem of the pulmonary artery. J Cardiovasc Electrophysiol. 2002;13:281-284. PubMed PMID. 11942598. Epub 2002/04/11. Eng.

7. Sekiguchi Y, Aonuma K, Takahashi A, et al. Electrocardiographic and electrophysiologic characteristics of ventricular tachycardia originating within the pulmonary artery. J Am Coll Cardiol. 2005;45:887-895. PubMed PMID. 15766825.

8. Iwai S, Cantillon DJ, Kim RJ, et al. Right and left ventricular outflow tract tachycardias: evidence for a common electrophysiologic mechanism. J Cardiovasc Electrophysiol. 2006;17:1052-1058. PubMed PMID. 16800855. Epub 2006/06/28. Eng.

9. Ya J, van den Hoff MJ, de Boer PA, et al. Normal development of the outflow tract in the rat. Circ Res. 1998;82:464-472. PubMed PMID. 9506707. Epub 1998/03/20. Eng. 
10. Hasdemir C, Aktas S, Govsa F, et al. Demonstration of ventricular myocardial extensions into the pulmonary artery and aorta beyond the ventriculo-arterial junction. Pacing Clin Electrophysiol. 2007;30:534539. PubMed PMID. 17437578. Epub 2007/04/18. Eng.

11. Liu CF, Cheung JW, Thomas G, et al. Ubiquitous myocardial extensions into the pulmonary artery demonstrated by integrated intracardiac echocardiography and electroanatomic mapping: changing the paradigm of idiopathic right ventricular outflow tract arrhythmias. Circ Arrhyth Electrophysiol. 2014;7:691-700. PubMed PMID. 24917663. Epub 2014/06/12. Eng.

12. Liao Z, Zhan X, Wu S, et al. Idiopathic ventricular arrhythmias originating from the pulmonary sinus cusp: prevalence, electrocardiographic/electrophysiological characteristics, and catheter ablation. J Am Coll Cardiol. 2015;66:2633-2644. PubMed PMID. 26670064

13. Yang L, Sun G, Chen X, et al. Meta-analysis of zero or near-zero fluoroscopy use during ablation of cardiac arrhythmias. Am J Cardiol. 2016;118:1511-1518. PubMed PMID. 27639689. Epub 2016/09/19. Eng.

14. Pass RH, Gates GG, Gellis LA, et al. Reducing patient radiation exposure during paediatric SVT ablations: use of CARTO(R) 3 in concert with "ALARA" principles profoundly lowers total dose. Cardiol Young. 2015;25:963-968. PubMed PMID. 25155609. Epub 2014/08/27. Eng.

15. Acosta J, Penela D, Herczku C, et al. Impact of earliest activation site location in the septal right ventricular outflow tract for identification of left vs right outflow tract origin of idiopathic ventricular arrhythmias. Heart Rhythm. 2015;12:726-734. PubMed PMID. 25542998. Epub 2014/12/30. Eng.

16. Dixit S, Gerstenfeld EP, Callans DJ, et al. Electrocardiographic patterns of superior right ventricular outflow tract tachycardias: distinguishing septal and free-wall sites of origin. J Cardiovasc Electrophysiol. 2003;14:1-7. PubMed PMID. 12625602. Epub 2003/03/11. Eng.

How to cite this article: Bansal N, Kobayashi D, Karpawich PP. Pulmonary damage following right ventricular outflow tachycardia ablation in a child: When electroanatomical mapping isn't good enough. Pacing Clin Electrophysiol. 2018;41:561-565. https://doi.org/10.1111/pace.13236 